\title{
Utilización de glicerina USP durante una restricción energética temprana en pollos de engorde
}

\author{
Avellaneda, Y. ${ }^{@} ;$ Afanador, G. ${ }^{2}$ y Ariza-Nieto, C.J. ${ }^{1}$
}

'Corporación colombiana de Investigación Agropecuaria, Agrosavia. Mosquera. Colombia.

${ }^{2}$ Facultad de Medicina Veterinaria y de Zootecnia. Universidad Nacional. Bogotá. Colombia.

\section{Palabras ClaVe adicionales}

Crecimiento compensatorio.

Desempeño productivo.

Temperatura rectal.

\section{RESUMEN}

En este estudio se evalúo el efecto de la inclusión de diferentes niveles de glicerina USP en dietas de pollos de engorde sometidos a una restricción energética del $85 \%$ del día 5 al 21 de edad. Los pollos de engorde recibieron dietas durante el periodo de restricción con inclusión de 0 , $2.5,5.0$ o $7.5 \%$ de glicerina USP. Los tratamientos contaron con 6 repeticiones y 26 pollos por repetición. Diariamente, se determinó la temperatura rectal de dos pollos por repetición. Semanalmente, se midió el peso corporal y los residuales de alimento, lo que permitió calcular el consumo y la conversión alimenticia. Las aves fueron beneficiadas al día 42. La información se analizó a través de un diseño completamente al azar con arreglo factorial $4 \times 2$. Los pollos de engorde restringidos energéticamente depositaron más grasa abdominal $(P<0.05)$ y no lograron alcanzar el peso corporal de los pollos alimentados a voluntad, sin embargo, registraron una menor $(P<0.05)$ conversión alimenticia y relación ventrículo derecho a peso total ventricular (VD/PTV). La inclusión de glicerina USP en las dietas de iniciación afectó linealmente $(P<0.05)$ el momento en que los pollos de engorde lograron estabilizar la temperatura corporal. Además, la conversión alimenticia y el rendimiento en pechuga tendieron $(\mathrm{P}<0.1)$ a mejorar con inclusiones entre 2.5 y $5.0 \%$ de glicerina USP, durante la fase de iniciación. Finalmente, se observó que, dentro del grupo de pollos alimentados a voluntad, los que consumieron dietas con 5 o $7.5 \%$ de glicerina USP registraron un mayor beneficio económico. En conclusión, la utilización de glicerina USP en pollos de engorde alimentados a voluntad mejora la respuesta productiva y económica.

\section{USP glycerin utilization during early energy restriction in broilers}

\section{SUMMARY}

\section{ADDITIONAL KEYWORDS}

Compensatory growth.

Productive performance

Rectal temperature.

INFORMATION

Cronología del artículo.

Recibido/Received: 15.06.2017

Aceptado/Accepted: 01.09.2018

On-line: 15.10 .2018

Correspondencia a los autores/Contact e-mail:

yavellaneda@agrosavia.co

\section{INTRODUCCIÓN}

En Colombia, el consumo de pollo ha incrementado en los últimos años, registrando un crecimiento del 13.3\% entre los años 2012 y 2013 (FENAVI, 2016). Por otro lado, los sistemas de producción de pollo de engorde establecidos en el piso térmico frío (superior a $2500 \mathrm{msnm}$ ) utilizan esquemas de restricción alimen- ticia para disminuir la incidencia de ascitis por hipoxia e incrementar la eficiencia de productiva (Uribe 2011).

Históricamente se considera que la restricción alimenticia es una estrategia que incrementa la productividad de la industria avícola (Sahraei 2013, p. 202) al mejorar las tasas de crecimiento (Govaerts et al. 2000, p. 359; Santoso 2002, p. 1322) y hacer más eficiente el sistema de alimentación (Plavnik y Hurwitz 1991, p. 
348; Tůmová et al. 2002, p. 426); sin embargo, en las últimas investigaciones reportadas en la literatura, la eficiencia de este proceso no arroja resultados positivos concluyentes (Azis et al. 2011, p. 53; Fanooci y Torki 2010, pp. 278-9; Mohebodini et al. 2009, p. 2072; Ozkan et al. 2010, pp. 979-81; Zhan et al. 2007, p. 656), lo que sugiere que las estirpes modernas exhiben un patrón de crecimiento compensatorio diferente, que debe ser estudiado con estrategias de alimentación particulares para cada estirpe.

Con el incremento en la producción mundial de biodiesel se ha incrementado exponencialmente la oferta de glicerina, el principal coproducto de la producción de este biocombustible (Thompson y He 2006, p. 261), y disminuido el precio en el mercado (por ejemplo, el precio de la glicerina USP se redujo en $77.5 \%$ entre el 2000 y el 2010 (Ciriminna et al. 2014, p. 5)). La anterior situación, junto al hecho de que la utilización no afecta la salud de animal o la calidad de la carne (Beserra, Cesar y Peres 2016, p. 264), posibilita el uso de este coproducto como un recurso energético alternativo en sistemas de alimentación animal (Bordignon et al. 2017; Lokesha et al. 2017; Silva et al. 2014). Además, el glicerol en monogástricos es absorbido a nivel intestinal en altas cantidades (Kato et al. 2004, pp. 1827-30), debido al bajo peso molecular y a que la absorción se realiza a través de sistemas pasivos (Emmanuel, Berzins y Robblee 1983, p. 566), constituyéndose así en un recurso con alto valor de energía efectiva (Emmans, 1994, p. 804).

Los estudios realizados en alimentación de aves han concluido que la pureza de la glicerina es un determinante en el nivel de utilización (Jung and Batal 2011, p. 526; Souza et al. 2017, p. 625). En ese sentido, en los estudios iníciales con pollos de engorde se utilizó glicerol de alta pureza y se observó una respuesta productiva óptima (Simon et al. 1996, pp. 108-10), posteriormente, se han usado glicerinas con diferente nivel de pureza y se ha observado un efecto positivo tanto al comienzo (Abd-Elsameeet al. 2010, p. 291; McLea et al. 2011, p. 372; Silva et al. 2012, p. 199) como al final del periodo de engorde (Avellaneda et al. 2009, p. 3; da Silva et al. 2017, p. 2087).

Dada la respuesta diferenciada a la restricción alimenticia y la posibilidad de usar glicerina, que ha demostrado atenuar el esfuerzo cardiaco en humanos (Anderson et al. 2001, p. 331; Coutts et al. 2002, pp. 116-7), se planteó este estudio para evaluar el efecto de la inclusión de glicerina USP en dietas de pollos de engorde sometidos a una restricción energética leve (85\% del consumo energético a voluntad), del día 5 al 21 de edad, en condiciones de altitud.

\section{MATERIALES Y MÉTODOS}

\section{UNIDADES EXPERIMENTALES}

Para este estudio se utilizaron 1248 pollos de engorde de la estirpe ROSS, de un día de edad, criados a una densidad inicial de 52 aves por corral $\left(20\right.$ aves $\left./ \mathrm{m}^{2}\right)$, disminuyéndose, después del día 21 de edad, a 25 pollos por corral $\left(9.6\right.$ aves $\left./ \mathrm{m}^{2}\right)$, considerando 6 repeticiones por tratamiento y 26 aves por repetición.

\section{AvAl DE COMITÉ DE BIOÉTICA}

Los protocolos de manejo animal fueron avalados por el comité de Bioética de la Facultad de Medicina Veterinaria y de Zootecnia, de la Universidad Nacional de Colombia, sede Bogotá (acta 05).

\section{UBICACIÓN}

Este estudio se realizó en la Unidad de Avicultura del CI Tibaitatá de la Corporación Colombiana de Investigación Agropecuaria, Agrosavia, ubicada a 2516 msnm (4.685222 -74.204722).

\section{GLICERINA}

Se utilizó glicerina USP (4208 kcal/ kg de EB, 1.1\% de humedad, $95.7 \%$ de glicerol, 0.8 ppm de metanol) para evitar efectos de otros componentes que contienen algunas glicerinas crudas. Se usó un valor de $4039 \mathrm{kcal}$ de EMAn $/ \mathrm{kg}$, obtenido en un experimento previo.

\section{DiETAS EXPERIMENTALES}

Se diseñaron dos dietas, una con concentraciones normales de nutrientes no calóricos, que se suministró a los pollos no restringidos, y una concentrada en nutrientes no-calóricos, para los pollos correspondientes a los tratamientos restringidos, buscando reducir el consumo de energía. En dietas de cada grupo se incluyó la glicerina USP en concentraciones de 0.0, 2.5, 5.0 y 7.5\%. Las dietas fueron constituidas básicamente por soya y maíz, como se presenta en la Tabla I.

Después del periodo de restricción (día 22 de edad), los pollos de todos los grupos experimentales recibieron alimento tipo comercial de engorde (3100 Kcal de $\mathrm{EM} / \mathrm{Kg}, 19.5 \%$ de PC, $1.0 \%$ de lisina digestible, $0.86 \%$ de Ca) hasta el día 35 y finalización (3150 Kcal de EM/ $\mathrm{Kg}, 18.5 \%$ de $\mathrm{PC}, 0.95 \%$ de lisina digestible, $0.76 \%$ de Ca) hasta el día 42.

\section{INSTALACIONES Y MANEJO ANIMAL}

El bioensayo se llevó a cabo en corrales en piso con cama de cascarilla de arroz. La temperatura inicial fue de $34^{\circ} \mathrm{C}$ y se realizó una reducción semanal de $3^{\circ} \mathrm{C}$, terminando en una temperatura que oscilaba entre 18 y $23^{\circ} \mathrm{C}$. La iluminación para la primera semana fue continua y a partir de la segunda semana se contó con 16 horas de luz (4 iluminación artificial) y 8 de oscuridad.

\section{PROTOCOLO EXPERIMENTAL}

Los pollos de engorde, durante los cuatro primeros días del ciclo, tuvieron acceso sin restricción al alimento, luego de este tiempo, a los grupos restringidos se les ofreció el 85\% del consumo que reportó una simulación con datos históricos de la unidad, el cual se ajustó diariamente según el nivel de alimento residual que se midió en los comederos. Se consideró cómo variable de madurez fisiológica la regulación de la temperatura rectal de los pollos de engorde, para ello, se tomaron de cada repetición dos pollos de engorde marcados y se les hizo seguimiento diario del peso corporal y la temperatura rectal (TR), hasta el día 21 de edad, esta última variable se ajustó a dos tipos de modelos: Lineal $(\mathrm{TR})=$ asíntota $+\mathrm{k}^{*}(\text { Plateau }- \text { edad })^{*} 1$, si edad $<$ Plateau, 0 ; Cuadrático $(\mathrm{TR})=$ asíntota $+\mathrm{k}^{*}\left((\text { Plateau }- \text { edad })^{2}\right)^{*} 1$, si edad $<$ Plateau, 0 . 
Tabla I. Dietas experimentales usadas para determinar el efecto de incluir glicerina USP en dietas de pollos alimentados a voluntad o restringidos energéticamente (Experimental diets used to determine the effect of including glycerin USP in diets of chickens fed at will or energy restricted).

\begin{tabular}{|c|c|c|c|c|c|c|c|c|}
\hline \multirow[b]{2}{*}{ Ingredientes (\%) } & \multicolumn{4}{|c|}{ Dietas a voluntad } & \multicolumn{4}{|c|}{ Dietas restricción } \\
\hline & $\begin{array}{c}\text { Glicerina } \\
0.0 \%\end{array}$ & $\begin{array}{c}\text { Glicerina } \\
2.5 \%\end{array}$ & $\begin{array}{c}\text { Glicerina } \\
5.0 \%\end{array}$ & $\begin{array}{c}\text { Glicerina } \\
7.5 \%\end{array}$ & $\begin{array}{c}\text { Glicerina } \\
0.0 \%\end{array}$ & $\begin{array}{c}\text { Glicerina } \\
2.5 \%\end{array}$ & $\begin{array}{c}\text { Glicerina } \\
5.0 \%\end{array}$ & $\begin{array}{c}\text { Glicerina } \\
7.5 \%\end{array}$ \\
\hline Maíz & 56.83 & 54.38 & 51.30 & 46.88 & 53.18 & 50.51 & 47.84 & 45.17 \\
\hline Glicerina USP & 0.00 & 2.50 & 5.00 & 7.50 & 0.00 & 2.50 & 5.00 & 7.50 \\
\hline Harina de arroz & 8.00 & 8.00 & 8.00 & 8.00 & 0.00 & 0.00 & 0.00 & 0.00 \\
\hline Torta de soya 45 & 29.15 & 29.41 & 30.24 & 32.37 & 38.90 & 39.35 & 39.80 & 40.25 \\
\hline Harina de pescado & 1.50 & 1.50 & 1.50 & 1.50 & 1.50 & 1.50 & 1.50 & 1.50 \\
\hline Aceite de soya & 0.53 & 0.22 & 0.00 & 0.00 & 1.61 & 1.32 & 1.04 & 0.76 \\
\hline Sal $(\mathrm{NaCl})$ & 0.30 & 0.30 & 0.30 & 0.30 & 0.35 & 0.35 & 0.35 & 0.35 \\
\hline Bicarbonato de sodio & 0.33 & 0.33 & 0.30 & 0.20 & 0.48 & 0.47 & 0.46 & 0.45 \\
\hline Carbonato de $\mathrm{Ca}$ & 0.93 & 0.92 & 0.91 & 0.88 & 1.02 & 1.01 & 1.00 & 0.99 \\
\hline Fosfato monobicálcico & 1.18 & 1.20 & 1.23 & 1.25 & 1.59 & 1.61 & 1.64 & 1.66 \\
\hline DL-Metionina & 0.20 & 0.20 & 0.20 & 0.19 & 0.24 & 0.25 & 0.25 & 0.26 \\
\hline HCl Lisina & 0.27 & 0.26 & 0.24 & 0.18 & 0.24 & 0.24 & 0.23 & 0.22 \\
\hline L-Treonina & 0.11 & 0.11 & 0.11 & 0.08 & 0.11 & 0.11 & 0.11 & 0.11 \\
\hline Cloruro de colina $60 \%$ & 0.07 & 0.07 & 0.07 & 0.07 & 0.08 & 0.08 & 0.08 & 0.08 \\
\hline Premezcla vit y min $^{*}$ & 0.60 & 0.60 & 0.60 & 0.60 & 0.70 & 0.70 & 0.70 & 0.70 \\
\hline \multicolumn{9}{|l|}{ Análisis calculado } \\
\hline EM (Mcal/Kg) & 2950 & 2950 & 2950 & 2950 & 2950 & 2950 & 2950 & 2950 \\
\hline Proteína cruda (\%) & 21.4 & 21.3 & 21.2 & 21.6 & 24.7 & 24.7 & 24.7 & 24.7 \\
\hline Calcio (\%) & 0.89 & 0.89 & 0.89 & 0.89 & 1.05 & 1.05 & 1.05 & 1.05 \\
\hline Fósforo disponible (\%) & 0.45 & 0.45 & 0.45 & 0.45 & 0.53 & 0.53 & 0.53 & 0.53 \\
\hline Lisina digestible (\%) & 1.21 & 1.21 & 1.21 & 1.21 & 1.41 & 1.41 & 1.41 & 1.41 \\
\hline
\end{tabular}

*Premezcla de vitaminas y minerales (aporte por kilogramo de dieta): vitamina A, 8500 IU; vitamina D3, 3200 IU; vitamina E, 32 IU; vitamina K3, $2.0 \mathrm{mg}$; tiamina, $4.0 \mathrm{mg}$; riboflavina, $5.5 \mathrm{mg}$; niacina, $42 \mathrm{mg}$; ácido pantoténico, $15 \mathrm{mg}$; piridoxina, $4.2 \mathrm{mg}$; biotina, $0.1 \mathrm{mg}$; ácido fólico, $1.1 \mathrm{mg}$; vitamina B12, $0.02 \mathrm{mg}$; colina, 1,1 mg; Zn, 80 mg; Mn,70 mg; Fe, 55 mg; Cu, 12mg; I, 1.1 mg; Se, 0.4 mg.

Durante el experimento se realizaron pesajes semanales del grupo de pollos de cada repetición y se midió el residual de alimento. Al día 42 de edad se tomaron dos pollos, con el peso promedio de la repetición, y se beneficiaron en una línea de sacrificio comercial, para reportar el rendimiento en canal caliente y el peso de cada una de las fracciones. De los pollos de engorde que murieron durante el experimento y los que se sacrificaron para determinar el rendimiento de las fracciones se obtuvo el corazón, el cual fue preservado en formalina hasta el final del ensayo, momento en el que se diseccionó el ventrículo derecho y se relacionó con el peso total ventricular (VD/PTV o índice cardiaco, IC), como un indicador de incidencia de ascitis. El pesaje se realizó en balanza analítica con sensibilidad de $0.01 \mathrm{~g}$.

\section{ANÁLISIS ESTADÍSTICO}

La información se analizó a través diseño completamente al azar con arreglo factorial $4 \times 2$, considerando los cuatro niveles de glicerina USP en las dietas y las dos ofertas energéticas. Los efectos principales se evaluaron por medio de una prueba de Tukey y la interacción por medio de una prueba de medias ajustadas. Adicionalmente, se evaluó el tipo de efecto (lineal, cuadrático o cúbico) de la inclusión de glicerina en la dieta. Para determinar el momento en que la temperatura rectal de los pollos de engorde llegó a una asíntota, se ajustaron dos tipos de modelos de broken line, uno de fase ascendente lineal y uno de fase ascendente cuadrática, con el procedimiento NLIN de SAS®. La información fue procesada a través del paquete estadístico $\mathrm{SAS}{ }^{\circledR}$ versión 9.2 , con el procedimiento GLM.

\section{RESULTADOS Y DISCUSIÓN}

De los modelos matemáticos utilizados para estimar la temperatura rectal de los pollos de engorde, el que mejor ajuste presentó fue el modelo de crecimiento inicial cuadrático respecto al lineal ( $R^{2}: 0.51$ vs 0.44 y CME: 0.298 vs 0.345$)$. Por otro lado, los pollos de engorde en este experimento ajustaron rápidamente la temperatura corporal, ya que al día 8 de edad la población alcanzó un valor superior a $41^{\circ} \mathrm{C}$, mientras que, a nivel general se considera que la temperatura rectal se estabiliza entre los días 10 y 15 días de edad (Furlan y Macari 2002, p. 215). Sin embargo, se reconoce que los pollos de engorde criados a bajas temperaturas aumentan la tasa metabólica para poder ajustarse y termorregular más rápidamente (Furlan y Macari, 2002, p. 215), lo que podría explicar la tendencia observada. 


\begin{tabular}{|c|c|c|c|c|}
\hline Consumo de energía & Glicerina (\%) & Máximo $\left({ }^{\circ} \mathrm{C}\right)$ & Tasa Constante & Edad al plateau (d) \\
\hline \multirow[t]{4}{*}{ A Voluntad } & 0.0 & 41.0 & -0.093 & 7.3 \\
\hline & 2.5 & 41.1 & -0.057 & 9.5 \\
\hline & 5.0 & 41.1 & -0.040 & 11.5 \\
\hline & 7.5 & 41.1 & -0.055 & 9.7 \\
\hline \multirow[t]{4}{*}{ Restringido } & 0.0 & 40.9 & -0.112 & 5.5 \\
\hline & 2.5 & 41.0 & -0.051 & 9.3 \\
\hline & 5.0 & 40.9 & -0.040 & 7.8 \\
\hline & 7.5 & 41.1 & -0.019 & 12.2 \\
\hline EEM & & 0.032 & 0.019 & 1.536 \\
\hline \multicolumn{5}{|l|}{ Efecto $^{1}$} \\
\hline Restricción energética & & NS & NS & NS \\
\hline Glicerina & & NS & * & NS \\
\hline Tipo de efecto & & NS & Lineal & Lineal \\
\hline Consumo*Glicerina & & NS & NS & NS \\
\hline
\end{tabular}

La restricción alimenticia no afectó $(\mathrm{P}>0.05)$ ninguno de los parámetros de la modelación de la temperatura rectal. El anterior efecto también fue reportado por Olukomaiya et al. (2015, p. 69), quienes evaluado dos estrategias de restricción no encuentran diferencia en la temperatura rectal al final del periodo de restricción (día 35 de edad), sin embargo, otros autores utilizan la estrategia de la restricción alimenticia para lograr que los pollos se adapten al estrés calórico (Abioja et al. 2014, p. 316), logrando que su temperatura rectal sea menor. Por otro lado, la inclusión de glicerina USP en las dietas de iniciación disminuyó linealmente la tasa constante del modelo cuadrático seleccionado (Tabla
II), lo que hizo que la edad a la cual se estabiliza la temperatura rectal fuera mayor al incluir glicerina en la dieta. El resultado de la glicerina sobre la temperatura rectal puede ser consecuencia del efecto termorregulador observado en atletas humanos hiperhidratados con glicerina (Easton et al. 2007, p. 81; Lyons et at. 1990, pp. 480-3), en donde el uso de este coproducto incrementa la tasa de sudor y la temperatura externa, efectos que se asocian con el incremento en el flujo sanguíneo periférico, lo que podría aumentar la disipación de calor vía radiación, conducción y conexión (van Rosendal et al. 2009, p. 698; Anderson et al. 2001, pp. 324-9).

Tabla III. Desempeño durante la fase de iniciación de pollos de engorde restringidos en energía y alimentados con diferentes niveles de glicerina USP (Performance during the initiation phase of broilers restricted in energy and fed with different levels of glycerin USP).

\begin{tabular}{|c|c|c|c|c|c|}
\hline Consumo & Glicerina (\%) & Ganancia de peso $(\mathrm{g} / \mathrm{d})$ & Conversión alimenticia & Homogeneidad (\%) & Supervivencia (\%) \\
\hline \multirow{4}{*}{ A voluntad } & 0.0 & 30.1 & 1.63 & 10.3 & 94.8 \\
\hline & 2.5 & 29.7 & 1.66 & 12.2 & 95.5 \\
\hline & 5.0 & 29.4 & 1.67 & 12.8 & 97.4 \\
\hline & 7.5 & 29.5 & 1.67 & 9.6 & 98.1 \\
\hline \multirow{4}{*}{ Restringidos } & 0.0 & 26.7 & 1.55 & 10.7 & 95.7 \\
\hline & 2.5 & 26.4 & 1.57 & 11.6 & 98.3 \\
\hline & 5.0 & 26.6 & 1.56 & 10.3 & 97.5 \\
\hline & 7.5 & 25.4 & 1.63 & 10.7 & 99.4 \\
\hline EEM & & 0.377 & 0.011 & 0.368 & 0.84 \\
\hline \multicolumn{6}{|l|}{ Efecto $^{1}$} \\
\hline Consumo de energía & & $* *$ & $* *$ & NS & * \\
\hline Glicerina & & NS & * & NS & * \\
\hline Tipo de efecto & & Lineal & Lineal & Cuadrático & Lineal \\
\hline Consumo*Glicerina & & NS & NS & NS & NS \\
\hline
\end{tabular}


Los pollos de engorde alimentados a voluntad ganaron $3.2 \mathrm{~g} / \mathrm{d}$ más $(\mathrm{P}<0.05)$ de peso corporal frente al grupo restringido, durante la fase de iniciación (Tabla III). Sin embargo, la conversión alimenticia de los pollos de engorde restringidos fue menor $(\mathrm{P}<0.05)$ en $4.2 \%$ frente al grupo control y tuvieron $1.3 \%$ más sobrevivencia. Por otro lado, los pollos de engorde que recibieron $7.5 \%$ de glicerina tuvieron una mayor $(\mathrm{P}<0.05)$ conversión alimenticia comparada con el grupo control, pero registraron $(\mathrm{P}<0.05) 2.9 \%$ menos de mortalidad que los pollos del grupo control o alimentados con $5 \%$ de glicerina.

El efecto negativo de la inclusión de glicerina sobre la conversión alimenticia en la fase de iniciación contrasta con trabajos como el de Mandalawi et al. (2014, p. 2859) quienes reportan un efecto positivo sobre esta variable usando dietas con glicerina cruda hasta el $10 \%$, o el de McLea et al. (2011, p. 372) quienes observaron mejora de un $11 \%$ al consumir dietas con $6.7 \%$ de glicerina en pollos de engorde jóvenes.

El nivel de restricción energética afectó $(\mathrm{P}<0.05)$ el consumo de alimento de la fase de engorde (Tabla IV), ya que las aves que fueron restringidas durante el periodo de iniciación consumieron $2 \mathrm{~g}$ menos por día. Sin embargo, cuando se expresa el consumo en función del peso corporal ( $\mathrm{g}$ de alimento/g de peso corporal), se observa que los pollos de engorde restringidos consumieron $4 \%$ más alimento $(\mathrm{P}<0.05)$ y ganaron $6 \%$ más de peso ( $\mathrm{g}$ de ganancia/g de peso vivo) $(\mathrm{P}<0.05)$. La conversión alimenticia de la fase de engorde, asociada al nivel de inclusión de glicerina en la dieta durante la fase de iniciación, exhibió un efecto cuadrático (con un mínimo de conversión estimado cuando se incluye $4 \%$ de glicerina en la dieta).

La respuesta cuadrática de algunas variables productivas frente a la inclusión de glicerina coincide con varios reportes de literatura, como el de McLea et al. (2011, p. 375$)$ con un óptimo cercano a $6.7 \%$ de inclu- sión, el de Avellaneda et al. (2009, p. 3), en condiciones de altitud, de 3.9\% para minimizar conversión alimenticia, el de Sehu et al. (2012, p. 197) con un óptimo alrededor de $5 \%$ de inclusión y al reporte de Jung y Batal, (2011, p. 524) donde una inclusión de $2.5 \%$ de glicerina maximiza la eficiencia alimenticia en el periodo de 0 a 14 días de edad. De esta manera, como lo muestra Romano et al. (2014, p. 101), existe un nivel de saturación de toma de glicerol que podría corresponder a $7.5 \%$, ya que cuando se usan dietas de $10 \%$, el nivel de glicerol en sangre se incrementa como un indicativo de la incapacidad para retenerlo en las células.

Los pollos de engorde restringidos energéticamente pesaron $76 \mathrm{~g}$ menos $(\mathrm{P}<0.05)$, registraron $0.05 \mathrm{~g} / \mathrm{g}$ menos $(\mathrm{P}<0.05)$ de conversión alimenticia y presentaron un menor IC al terminar el ciclo de producción comparado con aquellos alimentados a voluntad (Tabla V). Por otro lado, dentro del grupo de pollos alimentados a voluntad se observó que el IC de los pollos que no consumieron glicerina fue mayor $(\mathrm{P}<0.05)$ comparado con los que recibieron 5.0 o $7.5 \%$ de este coproducto y se registró un efecto lineal positivo $(\mathrm{P}<0.05)$ del consumo de glicerina sobre la supervivencia de los pollos de engorde.

En este estudio, los pollos de engorde no lograron exhibir crecimiento compensatorio después del periodo de restricción energética, lo cual afectó significativamente el peso final de los mismos. Reportes experimentales de restricción energética temprana son limitados en la literatura, sin embargo, Yang et al (2015, p. 165) restringiendo al $85 \%$ el consumo de energía y Gratta et al. (2017, p. 313), ofreciendo el 80\% del consumo observado en aves alimentadas a voluntad, encontraron que los pollos restringidos fueron más pequeños al final del ciclo productivo. Otros autores también reportan menor peso final en aves restringidas (Jahanpour, Seidavi y Qotbi 2014, pp. 90-2; Jalal y Zacaria 2012, pp. 818-9; Omosebi et al. 2014, p. 615; Zhan et al.

Table IV. Ganancia de peso y consumo de alimento durante la fase de engorde de pollos restringidos durante el periodo inicial de crecimiento y alimentados con glicerina USP.

\begin{tabular}{|c|c|c|c|c|c|c|}
\hline $\begin{array}{l}\text { Consumo de } \\
\text { energía }\end{array}$ & Glicerina (\%) & $\begin{array}{c}\text { Ganancia de } \\
\text { peso }(\mathrm{g} / \mathrm{d})\end{array}$ & $\begin{array}{l}\text { Ganancia } \\
(\mathrm{g} / \mathrm{d} / \mathrm{g} P V)\end{array}$ & $\begin{array}{c}\text { Consumo de alimento } \\
(\mathrm{g} / \mathrm{d})\end{array}$ & $\begin{array}{c}\text { Consumo de alimento } \\
(\mathrm{g} / \mathrm{d} / \mathrm{g} \mathrm{PV})\end{array}$ & $\begin{array}{l}\text { Conversión ali- } \\
\text { menticia (g/g) }\end{array}$ \\
\hline \multirow{4}{*}{ A voluntad } & 0.0 & 74.4 & 0.052 & 144.5 & 0.101 & 1.94 \\
\hline & 2.5 & 74.8 & 0.052 & 141.6 & 0.099 & 1.90 \\
\hline & 5.0 & 75.3 & 0.053 & 140.5 & 0.099 & 1.87 \\
\hline & 7.5 & 71.9 & 0.051 & 143.2 & 0.102 & 2.00 \\
\hline \multirow{4}{*}{ Restringidos } & 0.0 & 74.3 & 0.054 & 143.7 & 0.105 & 1.93 \\
\hline & 2.5 & 75.6 & 0.055 & 140.3 & 0.103 & 1.86 \\
\hline & 5.0 & 73.1 & 0.054 & 139.3 & 0.104 & 1.91 \\
\hline & 7.5 & 72.8 & 0.052 & 138.3 & 0.105 & 1.90 \\
\hline SEM & & 0.477 & 0.00054 & 0.736 & 0.00136 & 0.011 \\
\hline \multicolumn{7}{|l|}{ Efecto $^{1}$} \\
\hline Consumo de energía & & NS & $* *$ & * & $* *$ & NS \\
\hline Glicerina & & NS & NS & NS & NS & * \\
\hline Tipo de efecto & & NS & NS & NS & Cuadrático & Cuadrático \\
\hline Consumo*Glicerina & & NS & + & NS & NS & NS \\
\hline
\end{tabular}




\begin{tabular}{|c|c|c|c|c|c|c|c|}
\hline Consumo & Glicerina (\%) & Peso final (g) & Conversión $(\mathrm{g} / \mathrm{g})$ & Supervivencia (\%) & IC & Costo Parcial (\$/ave) & ${ }^{2}$ INP (\$/ave) \\
\hline \multirow{4}{*}{ A Voluntad } & 0.0 & 2211 & 1.79 & 91.6 & 0.30 & 1.630 & 0.608 \\
\hline & 2.5 & 2209 & 1.77 & 91.6 & 0.28 & 1.641 & 0.633 \\
\hline & 5.0 & 2210 & 1.77 & 96.2 & 0.26 & 1.709 & 0.804 \\
\hline & 7.5 & 2153 & 1.83 & 96.7 & 0.26 & 1.727 & 0.720 \\
\hline \multirow{4}{*}{ Restringido } & 0.0 & 2146 & 1.74 & 93.7 & 0.25 & 1.587 & 0.675 \\
\hline & 2.5 & 2153 & 1.71 & 95.8 & 0.24 & 1.626 & 0.758 \\
\hline & 5.0 & 2107 & 1.73 & 95.5 & 0.26 & 1.667 & 0.665 \\
\hline & 7.5 & 2072 & 1.76 & 95.9 & 0.27 & 1.652 & 0.675 \\
\hline EEM & & 29.3 & 0.015 & 1.53 & 0.005 & 0.029 & 0.055 \\
\hline \multicolumn{8}{|l|}{ Efecto $^{1}$} \\
\hline Consumo & & ** & ** & NS & * & ** & NS \\
\hline Glicerina & & NS & * & + & NS & * & NS \\
\hline Tipo de efecto & & NS & Cuadrático & Lineal & NS & Lineal & NS \\
\hline Consumo*Glicerina & & NS & NS & NS & * & NS & + \\
\hline
\end{tabular}

${ }^{1}+\mathrm{P}=0.05-0.1 ;{ }^{*} \mathrm{P}<0.05 ;{ }^{* *} \mathrm{P}<0.001$; NS: No significativo. ${ }^{2}$ Ingreso Neto Parcial por venta en canal

2007, p. 656). La incapacidad de los pollos de engorde para compensar se puede atribuir a la duración del periodo de restricción, que fue de 17 días, ya que autores de trabajos iníciales sobre restricción alimenticia en aves, mencionan que periodos de restricción mayores a 12 días impiden que los pollos alcancen el peso de sus contrapartes alimentadas a voluntad (Zubair y Leeson 1996, p. 727).

La conversión alimenticia de los pollos, durante la fase de engorde, se vio beneficiada por el efecto de la restricción energética del día 4 al 21 de edad, respuesta que ha sido reportada por otros autores (Jahanpour, Seidavi y Qotbi 2014, p. 91; Trocino et al. 2015, p. 2999) y justificada a través de un ahorro en los requerimientos para mantenimiento que están asociados al tamaño corporal (Talpaz et al. 1988, p. 383). Sin embargo, en otros trabajos la reducción del crecimiento luego de un periodo de restricción viene acompañada de una ineficiencia alimenticia (Jalal y Zacaria 2012, pp. 818-9; Jang et al. 2009, p. 391; Varol Avsilar y Onbasilar 2016, p. 72).

En términos generales, se considera que pollos de engorde con un IC por arriba de 0.28 presentan ascitis (Wideman 2000, p. 24). En ese sentido, las aves alimentadas con dietas cuya inclusión de glicerina fue superior a $5.0 \%$ no presentaron alteración en su condición fisiológica, mientras que sus contrapartes presentaron ascitis. Lo anterior podría estar relacionado con los beneficios cardiovasculares que se han observado en humanos tras el consumo de glicerol en situaciones de alto gasto cardiaco (Montner, Stark y Riedesel 1996, pp. 30-2), como es el caso de atletas de alto rendimiento, asociado a una reducción en la tasa cardiaca, incremento del llenado cardiaco y mantenimiento del volumen plasmático (Easton et al. 2007, pp. 83-5; Goulet et al. 2008 , p. 267). Por otro lado, similar a lo encontrado en este estudio, Boostani et al (2010, p. 174), encontraron que los pollos de engorde restringidos presentaron me- nor hipertrofia ventricular, sin embargo, otros trabajos reportan que esa estrategia alimenticia no evitó que se alterara el valor de IC (Ozcan et al. 2010, p. 982; Saber 2016, p. 4).

En cuanto al rendimiento de las fracciones de la canal, se observó que los pollos de engorde restringidos presentaron un mayor $(\mathrm{P}<0.05)$ valor de grasa abdominal $(1.51 \%)$ frente a los alimentados a voluntad $(1.22 \%)$, mientras que no se observaron diferencias significativas para el rendimiento de las fracciones (en promedio: $68.4 \%$ de canal, $31.6 \%$ de pierna-pernil, $11.7 \%$ de ala-costilla y $19.5 \%$ de rabadilla), pero se observó un efecto cuadrático de la inclusión de glicerina en la dieta sobre el porcentaje de pechuga de la canal, correspondiendo a un máximo de rendimiento con un nivel de inclusión de 3.4\% de glicerina USP.

La implementación de la restricción alimenticia se ha propuesto como una metodología para reducir el contenido de grasa de la canal (Yang et al. 2009, p. 224; Omosebi et al. 2014, p. 616), sin embargo, en este estudio los pollos que fueron restringidos energéticamente exhibieron un mayor porcentaje de grasa abdominal comparado con el grupo control, coincidiendo con resultados de otros trabajos (Lippens et al. 2000, p. 349; Poltowicz, Nowak y Wojtysiak 2015, p. 23). Paralelamente, el porcentaje de grasa abdominal en este estudio no se vio afectado por el uso de la glicerina, similar al reporte que hace Sehu et al. (2012, p. 198), sin embargo, Lessard, Lefrancois y Bernier (1993, p. 541) encontraron un incremento en la grasa abdominal cuando se incluyó glicerina al 5\%. Las discrepancias en los resultados de la grasa abdominal generalmente están asociados a la sobreestimación del valor energético de este recurso alimenticio, pero en este trabajo el valor de EM se basó de un bioensayo de balance previo con el mismo lote de glicerina. A nivel general se ha encontrado que la inclusión de glicerina en las dietas no 
altera el rendimiento en canal (Sehu et al. 2012, p. 198), sin embargo, Cerrate et al., (2006, p. 1003) encontraron que al $2.5 \%$ de inclusión se incrementa el porcentaje de pechuga, similar al 3.4\% registrado en este estudio.

El costo de producción de los pollos restringidos fue $2.6 \%$ menor, comparado con el de los pollos alimentados a voluntad, este resultado es superior al reportado por Simeon $(2015$, p. 4$)$ de 1.22, al restringir pollos de engorde al $85 \%$ del consumo observado a voluntad. Por otro lado, la inclusión de glicerina generó una respuesta lineal en el costo por ave encasetada, debido al precio de la glicerina USP (0.87 USD/Kg). Sin embargo, el uso de $5 \%$ de este recurso en un esquema de alimentación a voluntad presentó un mejor beneficio económico (0.2 USD más por pollo encasetado), en virtud de que la supervivencia de este grupo fue $4.6 \%$ mayor y el rendimiento en canal se incrementó en $0.8 \%$, comparado con el grupo control a voluntad.

\section{CONCLUSIONES}

El consumo de dietas con 5 o $7.5 \%$ de glicerina durante la fase de iniciación en pollos de engorde alimentados a voluntad disminuyó la hipertrofia ventricular, lo que mejora la respuesta productiva en condiciones de altitud. La conversión alimenticia y el rendimiento en pechuga de pollos alimentados con o sin restricción alimenticia mejoran con inclusiones de glicerina cercanas al $4 \%$. Además, el uso de este coproducto parece regular los mecanismos de control de la temperatura corporal, lo que sugiere su evaluación en sistemas de producción de pollo de engorde en condiciones de estrés calórico.

\section{Agradecimientos}

Los autores agradecen a COLCIENCIAS y ECOPETROL por la financiación del proyecto "Utilización de glicerina y otros subproductos del proceso de producción de biodiesel en sistemas de alimentación de aves y cerdos" y a Bio D S.A. por la provisión de la glicerina utilizada en este estudio.

\section{BIBLIOGRAFIA}

Abd-Elsamee, M, Abdo, Z, Manylawi, M \& Salim, I 2010 'Use of crude glycerin in broiler diets', Egyptian Poultry Science, vol. 30, no. 1, pp. 281-95.

Abioja, MO, Sodipe, OG, Abiona, JA, Oladipo, KA, Kasali, OD, Akerele, Z, Ola, A, Oke, H, Ogundele, O and Osinowo, OA 2014 'Thermotolerance acquisition in broiler chickens through early feed restriction: response to acute heat stress', Pertanika Journal of Tropical Agricultural Science, vol. 37, no. 3, pp. 311-9.

Anderson, M. J. Cotter, A. Garnham, D. Casley and M. Febbraio, 2001. Effect of glycerol-induced hyperhydration on thermoregulation and metabolism during exercise in heat. International Journal of Sport Nutrition and Exercise Metabolism, vol. 11, no. 3, pp. 315-33.

Avellaneda, Y, Rodríguez, D, Afanador, G \& Ariza, C 2009 'Efecto de la inclusión de glicerina cruda sobre el desempeño productivo de hembras de pollos de engorde en la Sabana de Bogotá' Revista Colombiana de Ciencias Pecuarias, vol. 22, p. 3.

Azis, A, Abbasb, H, Heryandib,Y and Kusnadib, E 2011 'Compensatory growth and production efficiency of broiler chickens exposed to feeding time restriction' Media Peternakan, pp. 50-7.
Beserra, VA, Cesar, AS and Peres, AAC. 2016. Adocao da glicerina bruta na dieta animal e seu impacto no produto final. Archivos de Zootecnia, vol. 65, no. 250, pp. 259-66.

Boostani, A, Ashayerizadeh, A, Manmoodian, F and Kamalzadeh, A 2010 'Comparison of the effects of several feed restriction periods to control ascites on performance, carcass characteristics and hematological indices of broilers' Brazilian Journal of Poultry Science, vol. 12, no. 3, pp. 171-177.

Bordignon, G, Lima, HJD, Martins, RA and Fonseca, MM. 2017 'Niveis de glicerina bruta na racao de suinos nas fases de crescimento e terminacao' Archivos de Zootecnia, vol. 66, no. 255, pp. 429-32.

Cerrate, S, Yan, F, Wang, Z, Coto, C, Sacakli, P \&Waldroup, P 2006 'Evaluation of glycerine from biodiesel production as a feed ingredient for broilers' International Journal of Poultry Science, vol. 6, no. 11, pp. 1001-7.

Ciriminna, R, Della Pina, C, Rossi, M and Pagliaro, M 2014 'Understanding the glycerol market'European Journal of Lipid Science and Technology, vol. 116, no. 10, 1-8.

Coutts, A, Reaburn, P, Mummery, K and Holmes, M 2002 'The e区ect of glycerol hyperhydration on olympic distance triathlon performance in high ambient temperatures' International Journal of Sport Nutrition and Exercise Metabolism, vol. 12, pp. 105-19.

da Silva, MC, Vieira, RG, Rodrigues, KF, da Silva, GF, Sousa, LF, Rodrigues, FL, Alves, CF, Augusto, WF, Parente, IP, Bezerra, LS 2017 'Effects of purified glycerin in balanced diets of chicken broilers treated from 22 to 42 days of age' Semina: Ciências Agrárias, vol. 38, no. 4, pp. 2083-90.

Easton, C, Turner, S and Pitsiladis, Y 2007 'Creatine and glycerol hyperhydration in trained subjects before exercise in the heat' International Journal of Sport Nutrition and Exercise Metabolism, vol. 17, pp. 70-91.

Emmans, G 1994 'Effective energy: a concept of energy utilization applied across species' British Journal of Nutrition, vol. 71, pp. 801-21.

Emmanuel, B, Berzins, R and Robblee, A 1983 'Rates of entry of alanine and glycerol and their contribution to glucose synthesis in fasted chickens' British Poultry Science, vol. 24, no. 4, pp. 565-71.

Fanooci, M and Torki, M 2010 'Effects of Qualitative Dietary Restriction on Performance, Carcass Characteristics, White Blood Cell Count and Humoral Immune Response of Broiler Chicks' Global Veterinaria, vol. 4, no. 3, pp. 277-82

FENAVI, 2016 'Consumo percapita pollo en Colombia' Estadísticas Fenavi. Disponible en: http://www.fenavi.org/index.php?option=com_co ntent\&view $=$ article\&id $=2160$ \&ltemid $=556$.

Furlan, RLy Macari, M 2002 Capítulo 17: Termorregulaçao. En Fisiologia Aviaria, aplicada a frangos de corte. Macari M. Editora Funep. Sao Paulo. Brasil, pp. 375.

Goulet, E, Rousseau, S, Lamboley, C, Plante, G and Dionne, I 2008 'Preexercise hyperhydration delays dehydration and improves endurance capacity during $2 \mathrm{~h}$ of cycling in a temperate climate' Journal of Physiological Anthropology, vol. 27, no. 5, pp. 263-71.

Govaerts, T, Room, G, Buyse, J, Lippens, M, Groote, G and Decuypere, E 2000 'Early and temporary quantitative food restriction of broiler chickens. 2. Effects on allometric growth and growth hormone secretion', British Poultry Science, vol. 41, no. 3, pp. 355-62.

Gratta, F, Birolo, M, Xiccato, G and Trocino, A 2017 'Effect of genotype, gender, and feed restriction on slaughter results and meat quality of broiler chickens', Agriculturae Conspectus Scientificus, vol. 82, no. 3, pp. 311-4.

Jahanpour, H, Seidavi, A and Qotbi, AA 2014 'Effects of intensity and duration of quantitative restriction of feed on broiler performance' Journal of the Hellenic Veterinary Medical Society, vol. 65, no. 2, pp. 83-98.

Jalal, MA and Zakaria, HA 2012 'The effect of quantitative feed restriction during the starter period on compensatory growth and carcass characteristics of broiler chickens 'Pakistan Journal of Nutrition, vol. 11 , no. 9, pp. 817-22.

Jang, IS, Kang, SY, Ko, YH, Moon, YS and Sohn, SH 2009 'Effect of quantitative feed restriction on growth performance and immune func- 
tion in broiler chickens' Asian-Australasian Journal of Animal Sciences, vol. 22, no. 3, pp. 388-95

Jung, B and Batal, AB 2011 Nutritional and feeding value of crude glycerin for poultry. 2. Evaluation of feeding crude glycerin to broilers' Journal of Applied Poultry Research, vol. 20, pp. 514-27.

Kato, T, Hayashi, Y, Inoue K \& Yuasa, H 2004 'Function characterization of the carrier-mediated transport system for glycerol in everted sacs of the rat small intestine' Biological Pharmacology Bulletin, vol. 27, no. 11, pp. 1826-30.

Lessard, $P$, Lefrancois, MR and Bernier, JF 1993 'Dietary addition of cellular metabolic intermediates and carcass fat deposition in broilers' Poultry Science, vol. 72, pp. 535-45.

Lippens, M, Room, G, DeGroote, G and Decuypere, E 2000 'Early and temporary quantitative food restriction of broiler chickens. I. Effects on performance characteristics, mortality and meat quality' British Poultry Science, vol. 41, no. 3, pp. 343-354.

Lokesha, E, Gopi, M, Kumar, RD, Patel, B, Banakar, PS, Deginal, R, Pruthviraj, DR, Bhanuprakash, V and Mahesh, MS 2017 'Crude glycerol: by-product of biodiesel industries as an alternative energy source for livestock feeding' Journal of Experimental Biology and Agricultural Sciences, vol. 5, no. 6, pp. 755-766.

Lyons, T, Riedesel, M, Meuli, L and Chick, T 1990 'EXects of glycerol induced hyperhydration prior to exercise in the heat on sweating and core temperature' Medicine and Science in Sports and Exercise, vol. 22, no. 4, pp. 477-83.

Mandalawi, HA, Kimiaeitalab, MV, Obregon, V, Menoyo, D \&Mateos, GG 2014 'Influence of source and level of glycerin in the diet on growth performance, liver characteristics, and nutrient digestibility in broilers from hatching to 21 days of age' Poultry Science, vol. 93, pp. 2855-63.

McLea, L, Ball, M, Kilpatrick, D and Elliott, C 2011 'The effect of glycerol inclusion on broiler performance and nutrient digestibility' British Poultry Science, vol. 52, no. 3, pp. 368-75.

Mohebodini, H, Dastar, B, Shams, M and Zerehdaran, S 2009 'The comparison of early feed restriction and meal feeding on performance, carcass characteristics and blood constituents of broilers chickens' Journal of Animal and Veterinary Advances, vol. 8, no. 10, pp. 2069-74

Montner, P. Stark, D and Riedesel, M 1996 'Pre-exercise glycerol hydration improves cycling endurancetime' International Journal ofSports Medicine, vol. 17, no. 1, pp. 27-33.

Olukomaiya, OO, Adeyemi, OA, Sogunle, OM, Abioja, MO and Ogunsola IA 2015 'Effect of feed restriction and ascorbic acid supplementation on growth performance, rectal temperature and respiratory rate of broiler chicken', The Journal of Animal \& PlantSciences, vol. 25, no. 1, pp. 65-71.

Omosebi, DJ, Adeyemi, OA, Sogunle, MO, Idowu, OMO and Nioku, CP 2014 'Effects of duration and level of feed restriction on performance and meat quality of broiler chickens' Archivos de Zootecnia, vol. 63, no. 244, pp. $611-621$

Ozkan, S, Takma, C, Yahav, S, Soğut, B, Turkmut, L, Erturun, H and Cahaner, A 2010 'The effects of feed restriction and ambient temperature on growth and ascites mortality of broilers reared at high altitude' Poultry Science, vol. 89, pp. 974-85.

Plavnik, I and Hurwitz, S 1991 'Response of broiler chickens and turkey poults to food restriction of varied severity during early life', British Poultry Science, vol. 32, no. 2, pp. 343-52.

Połtowicz, K, Nowak, J and Woitysiak, D 2015 'Effect of feed restriction on performance, carcass composition and physicochemical properties of the m. pectoralis superficialis of broiler chickens', Annals of Animal Science, vol. 15, no. 4, pp. 1019-29.

Romano, GG, Menten, JFM, Freitas, LW, Lima, MB, Pereira, R, Zavarize, KC \& Dias CTS 2014 'Effects of glycerol on the metabolism of broilers FED increasing glycerine levels', Brazilian Journal of Poultry Science, vol. 16, no. 1, pp. 97-106

Saber, SN 2016 'Effect of quatitative feed restriction on carcass characteristics and some blood parameters in broilers chickens' International Research Journal of Biological Sciences, vol. 5, no. 10, pp. 1-6.
Sahraei, M 2013 'Improvement production efficiency and carcass quality through feed restriction programs in broiler chickens' Biotechnology in animal husbandry, vol. 29, no. 2, pp. 193-210.

Santoso, U 2002 'Effects of Early Feed Restriction on the Occurrence of Compensatory Growth, Feed Conversion Efficiency, Leg Abnormality and Mortality in Unsexed Broiler Chickens Reared in Cages', Asian-Australasian Journal of Animal Sciences, vol. 15, no. 9, pp. 1319-25.

SAS Institute, 2008. SAS Users Guide. SAS Institute Inc., Cary, NC.

Sehu, A, Kucukersan, S, Coskun, B, Koksal, BH \& Citil, OB 2012 'Effects of dietary glycerol addition on growth performance, carcass traits and fatty acid distribution in cloacal fat in broiler chickens', Revue de Médecine Vétérinaire, vol. 163, no. 4, pp.194-200.

Silva, CLS, Menten, JFM, Traldi, AB, Pereira, R, Zavarize, KC and Santarosa, J $2012^{\prime}$ Glycerine derived from biodiesel production as a feedstufffor broiler diets' Brazilian Journal of Poultry Science, vol. 14, no. 3, pp. 193-202.

Silva, VO, Lopes, E, Andrade, EF, Sousa, RV, Zangeronimo, MG \& Pereira, $\sqcup 2014$ 'Uso de co-productosdel biodiesel (Glicerol) como fuentes alternativas de energia em la alimentación animal: uma revisão sistemática', Archivos de Medicina Veterinaria, vol. 46, pp. 111-20.

Simeon, o 2015 'Effects of strain and feed restriction at starter phase on performance ofbroiler chickens in the humid tropics', International Journal of Agricultural Sciences and Natural Resources, vol. 2, no. 1, pp. 1-5.

Simon, A, Bergner, H \&Schwabe, M 1996 'Glycerol feed ingredient for broiler chickens' Archives of Animal Nutrition, vol. 49, pp. 103-12.

Souza, C, Nunes, RV, Broch, J and Wachholz, L. 2017. Producao e utilicao da glicerina bruta na alimentacao de frangos de corte. Archivos de Zootecnia, vol., 66, no. 256, pp. 619-27.

Talpaz, H, Hurtwitz, S, de la Torre, Jand Sharpe, P 1988 'Economic optimization of a growth trajectory for broilers' American Journal of Agricultural Economics, vol. 70, no. 2, pp. 382-390.

Thompson, J and He B 2006 'Characterization of crude glycerol from biodiesel production from multiple feedstocks', Applied Engineering in Agriculture, vol. 22, no. 2, pp. 261-65.

Trocino, A, Piccirillo, A, Birolo, M, Radaelli, G, Bertotto, D, Filiou, E, Petracci, $M$ and Xiccato, G 2015 'Effect of genotype, gender and feed restriction on growth, meat quality and the occurrence of white striping and wooden breast in broiler chickens', Poultry Science, vol. 94, pp. 2996-3004.

Tủmová, E, Skrìvan, M, Skřivanová, V and Kacerovská, L 2002 'Effect of early feed restriction on growth in broiler chickens, turkeys and rabbits', Czech Journal of Animal Science, vol. 47, no. 10, pp. 418-28.

Uribe, AJ, Valencia, JE, Morales, P, Triana, S y Velandia, D. 2011. Restricción alimenticia en pollos de engorda en explotaciones comerciales. XXII Congreso Latinoamericano de Avicultura, Buenos Aires, Argentina.

vanRosendal, S, Osborne, M, Fassett, R and Coombes, J 2009 'Physiological and performance e区ects of glycerol hyperhydration and rehydration', Nutrition Reviews, vol. 67, no. 12, pp. 690-705.

Varol Avcilar, O and Avcilar, EE 2016 'Effects of breeder age and early energy restriction on fattening performance, some meat quality traits and plasma leptin concentration of broilers', Ankara Üniversitesi Veteriner Fakültesi Dergisi, vol. 63, pp. 69-76.

Wideman, R 2000 'Cardio-pulmonary hemodynamics and ascites in broiler chickens' Avian and Poultry Biology Reviews, vol. 11, no. 1, pp. 24-43.

Yang, HY, Yang, Z, Wang, Z, Wang, W, Huang, K, Fan, W and Jia, T 2015 'Effects of early dietary energy and protein dilution on growth performance, nutrient utilization and internal organs of broilers' Italian Journal of Animal Science, vol. 14, no. 2, pp. 162-171.

Yang, YX, Guo, J, Yoon, SY, Jin, Z, Choi, JY, Piao, XS, Kim, BW, Ohh, SJ, Wang MH and Chae, BJ 2009 'Early energy and protein reduction: effects on growth, blood profiles and expression of genes related to protein and fat metabolism in broilers', British Poultry Science, vol. 50, no. 2, pp. $218-27$.

Zhan, X, Wang, M, Ren, H, Zhao, R, Li J and Tan, Z 2007 'Effect of early feed restriction on metabolic programming and compensatory growth in broiler chickens', Poultry Science, vol. 86, pp. 654-60.

Zubair, AK and Leeson, S 1996 'Changes in body composition and adipocyte cellularity of male broilers subjected to varying degrees of early-life feed restriction', Poultry Science, vol. 75, pp. 719-28. 